\title{
The rs61764370 Functional Variant in the KRAS Oncogene is Associated with Chronic Myeloid Leukemia Risk in Women
}

\author{
Humberto Gutiérrez-Malacatt ${ }^{1,4}$, Manuel Ayala-Sanchez ${ }^{2}$, Xochitl Aquino- \\ Ortega $^{2}$, Jacqueline Dominguez-Rodriguez ${ }^{2}$, Adolfo Martinez-Tovar ${ }^{3}$, Irma \\ Olarte-Carrillo ${ }^{3}$, Angelica Martinez-Hernandez ${ }^{4}$, Cecilia Contreras-Cubas $\mathbf{C}^{4}$, \\ Lorena Orozco $^{4}$, Emilio J Cordova ${ }^{4 *}$
}

\begin{abstract}
Background: Chronic myeloid leukemia (CML) is one of the most frequent hematopoietic malignancies in the elderly population; however, knowledge is limited regarding the genetic factors associated with increased risk for CML. Polymorphisms affecting microRNA (miRNA) biogenesis or mRNA:miRNA interactions are important risk factors in the development of different types of cancer. Thus, we carried out a case-control study to test the association with CML susceptibility of gene variants located in the miRNA machinery genes AGO1 (rs636832) and GEMIN4 (rs2740348), as well as in the miRNA binding sites of the genes BRCA1 (rs799917) and KRAS (rs61764370). Materials and Methods: We determined the genotype of 781 Mexican-Mestizo individuals (469 healthy subjects and 312 CML cases) for the four polymorphisms using TaqMan probes to test the association with CML susceptibility. Results: We found a borderline association of the minor homozygote genotype of the KRAS_rs61764370 polymorphism with an increased risk for CML susceptibility $(P=0.06)$. After gender stratification, this association was significant only for women (odds ratio $[O R]=13.41, P=0.04$ ). The distribution of the allelic and genotypic frequencies of the four studied SNPs was neither associated with advanced phases of CML nor treatment response. Conclusions: To the best of our knowledge, this study is the first to show a significant association of the KRAS_rs61764370 SNP with CML. To further determine such an association of with CML susceptibility, our results must be replicated in different ethnic groups.
\end{abstract}

Keywords: CML - polymorphisms - microRNAs - KRAS

Asian Pac J Cancer Prev, 17 (4), 2265-2270

\section{Introduction}

Chronic myeloid leukemia (CML) is a malignant myeloproliferative disease characterized by the reciprocal translocation $\mathrm{t}(9 ; 22)(\mathrm{q} 34 ; \mathrm{q} 11)$, which produces the fusion gene BCR-ABL1. The protein encoded by this fusion gene, named BCR-ABL, regulates several signal transduction pathways involved in cell adhesion, migration, apoptosis, and proliferation through its constitutive tyrosine kinase activity (Apperley, 2015). Although the advent of tyrosine kinase inhibitors has greatly improved CML treatment, the success of this therapy depends on early diagnosis of the disease (Radich, 2014; Ursan et al., 2015). Because most CML patients are asymptomatic during the first phase of the disease, the discovery of biomarkers suitable for the assessment of individual risk for developing CML in the healthy population is essential for reducing the morbidity and mortality associated with this illness.

MicroRNAs (miRNAs) are small non-coding RNAs (19-24 nt) involved in gene expression regulation through direct binding to their target messenger RNA (mRNA). The biosynthesis of miRNAs is initiated by their transcription as long RNA transcripts called pri-miRNAs (100 nt-10 kb), which are processed within the nucleus by a complex composed of the RNase III enzyme Drosha and its cofactor DGCR8 into 70 -nt precursors called premiRNAs. The pre-miRNAs are exported to the cytoplasm through the EXPO5 pathway and then processed by the multi-domain protein Dicer into 22-nt miRNA duplexes. These duplexes are loaded into Argonaute proteins in the RNA-induced silencing complex (RISC) as doublestranded RNA and subsequently unwound into mature, single-stranded molecules. The mature miRNA usually interacts with the 3' untranslated region (UTR) of the

${ }^{1}$ Posgrado en Ciencias Biologicas, Universidad Nacional Autonoma de Mexico, ${ }^{2}$ Service of Hematology, Hospital de Especialidades, CMN La Raza, IMSS, ${ }^{3}$ Department of Hematology, Hospital General de Mexico, Secretaría de Salud, ${ }^{4}$ Immunogenomics and Metabolic Diseases Laboratory, Instituto Nacional de Medicina Genomica, INMEGEN, SS, Mexico, D.F. Mexico *For correspondence: ecordova@inmegen.gob.mx 
target mRNA through a 2-8-nt sequence located at its 5, terminus called the "seed region" (Ha and Kim, 2014).

Recently, several studies have highlighted the importance of single nucleotide polymorphisms (SNPs) either in genes involved in miRNA biogenesis or inside miRNA binding sites (MBS) at the target mRNA for the risk of developing chronic-degenerative disorders. For instance, the rs636832 SNP located in the AGO1 gene (argonaute RISC catalytic component 1), which encodes the Argonaute protein that directly recruits miRNAs into the RISC complex, has been associated with a decreased risk for developing chronic hepatitis B and lung cancer (Kim et al., 2010; Shang et al., 2014). Likewise, the SNP rs2740348, located in the gene encoding the Gemin4 protein (GEMIN4 [gem (nuclear organelle) associated protein 4]), another important component of the RISC complex, has been correlated with a lower risk for prostate cancer development (Liu et al., 2012).

Regarding SNPs located within MBS at the target mRNAs, the minor allele of a SNP may alter the miRNA::mRNA interaction by abrogating or creating miRNA-binding sites, affecting the expression level of the target gene. Additionally, the rs61764370 variant, which has been associated with non-small cell lung cancer, is situated in the 3'-UTR region of the KRAS oncogene (Kirsten rat sarcoma viral oncogene homolog) and disrupts the binding of let-7 (Chin et al., 2008). Likewise, the rs799917 variant reduces the binding of miR-638 to the coding sequence of the tumor suppressor gene BRCA1 (Breast cancer 1, early onset) and has been associated with breast and esophageal carcinoma (Nicoloso et al., 2010; Zhang et al., 2013).

The involvement in CML susceptibility of SNPs occurring in genes related to the biogenesis of miRNAs or at MBS of a target mRNA, has been poorly studied until now. Therefore, the aim of this study was to determine the association of SNPs occurring in the miRNA processing genes AGO1 (rs636832) and GEMIN4 (rs2740348) and in MBS in the KRAS (rs61764370) and BRCA1 (rs799917) genes, with CML susceptibility.

\section{Materials and Methods}

\section{Study population}

This study included a total of 781 Mexican-Mestizos, including 312 individuals with CML (137 women and 175 men) and 469 healthy participants (206 women and 263 men). We also included 487 healthy unrelated individuals from four Amerindian groups (Maya, Nahuatl, Zapoteca, and Tarahumara). The CML-affected individuals were recruited at the service of hematology, in the "Hospital de Especialidades, CMN La Raza", and at the department of hematology, in the "Hospital General de México", both in Mexico City. CML diagnosis was confirmed in bone marrow aspirate by morphology, immunohistochemistry, cytogenetic and BCR/ABL detection by PCR. Samples from healthy Mexican-Mestizo individuals were collected at the blood bank from a tertiary hospital in Mexico City, whereas samples from individuals with Amerindian ancestry were collected at their locality of origin. Amerindian individuals identified themselves as indigenous, were born in the same area as their parents and grandparents, and had two parents or four grandparents who speak the native language. Cases and controls were matched by gender (male $56 \%$, female $44 \%$ in both groups) and age (cases: $44.87 \pm 14.88$; controls: $40.52 \pm 8.46$ ). This protocol was revised and accepted by local ethical and research committees and performed in accordance with the ethical standards of the 1964 Helsinki declaration and its later amendments. Additional informed consent was obtained from all individuals participants for whom identifying information is included in this article.

\section{SNP genotyping}

Genomic DNA was obtained from $10 \mathrm{~mL}$ of peripheral blood sample using a QIAamp DNA blood mini kit (QIAGEN, Hilden, Germany). Allelic discrimination of the SNPs rs636832 (AGO1), rs2740348 (GEMIN4), rs799917 (BRCA1), and rs61764370 (KRAS) was performed using TaqMan probes with an ABI 7900HT Real-Time PCR System (Applied Biosystems, Foster City, CA, USA). Detected genotypes were validated through direct sequencing of $10 \%$ of the analyzed samples using an ABI PRISM 3730 DNA analyzer.

\section{Statistical analysis}

Hardy-Weinberg equilibrium of the studied SNPs was evaluated with the Pearson's chi-square test with 1 degree of freedom. The allelic and genotypic frequencies were compared between CML and control subjects with a chi-square test, and association was determined by odds ratios (ORs). Statistical analysis was carried out using the bioinformatics tool Finetti (https://ihg.gsf.de/cgi-bin/hw/ hwa1.pl) and Epi info (v. 7.0). Results with a P value < 0.05 were considered statistically significant.

\section{Results}

Demographic data from CML patients and healthy individuals recruited to perform this study are shown in Table 1. The mean age of CML-affected individuals and healthy controls was $44.87 \pm 14.88$ and $40.52 \pm 8.46$, respectively. Most of the individuals with CML were diagnosed at the chronic phase of the disease $(0.83)$, followed by the blastic (0.09) and the accelerated phases (0.08).

After determining the allelic and genotypic frequencies of the SNPs AGO1_rs636832, GEMIN4_rs2740348,

Table 1. General Characteristics for the Chronic Myeloid Leukemia Cases and Control Samples

\begin{tabular}{lcc}
\hline & $\begin{array}{c}\text { Control } \\
\mathrm{n}=469\end{array}$ & $\begin{array}{c}\text { CML } \\
\mathrm{n}=312\end{array}$ \\
\hline Age (years) & $40.52 \pm 8.46$ & $44.87 \pm 14.88^{\mathrm{a}}$ \\
\hline Gender & & \\
Male & $263(56 \%)$ & $175(56 \%)$ \\
Female & $206(44 \%)$ & $137(44 \%)$ \\
& & Chronic (83\%) \\
CML Phase $^{\mathrm{b}}$ & $* * * * *$ & Accelerated (8\%) \\
& & Blast crisis (9\%) \\
\hline
\end{tabular}

${ }^{\mathrm{a}}$ Age at time of diagnosis; ${ }^{\mathrm{b}}$ At the time of diagnosis 
Table 2. Genotype and Allele Frequencies in CML Affected Individuals and Healthy Controls

\begin{tabular}{|c|c|c|c|c|c|}
\hline Gene & Genotype/ Allele & $\begin{array}{l}\text { Control } \\
\mathrm{n}=469 \\
\end{array}$ & $\begin{array}{c}\mathrm{CML} \\
\mathrm{n}=312\end{array}$ & OR $(95 \% \mathrm{CI})$ & Pvalue* \\
\hline \multirow{3}{*}{ AGO1 rs636832 } & AA & 0.26 & 0.23 & & \\
\hline & $\mathrm{AG}$ & 0.49 & 0.5 & $1.15(0.81-1.64)$ & 0.44 \\
\hline & GG & 0.25 & 0.27 & $1.21(0.81-1.81)$ & 0.35 \\
\hline \multirow{5}{*}{ GEMIN4 rs2740348 } & A & 0.5 & 0.48 & & \\
\hline & G & 0.49 & 0.52 & $1.10(0.90-1.35)$ & 0.35 \\
\hline & GG & 0.62 & 0.65 & & \\
\hline & GC & 0.35 & 0.3 & $0.84(0.61-1.14)$ & 0.26 \\
\hline & $\mathrm{CC}$ & 0.04 & 0.04 & $1.18(0.57-2.44)$ & 0.66 \\
\hline \multirow{5}{*}{ BRCA1 rs799917 } & $\mathrm{G}$ & 0.79 & 0.8 & & \\
\hline & $\mathrm{C}$ & 0.21 & 0.2 & $0.93(0.72-1.20)$ & 0.57 \\
\hline & $\mathrm{CC}$ & 0.43 & 0.47 & & \\
\hline & $\mathrm{CT}$ & 0.45 & 0.41 & $0.84(0.62-1.13)$ & 0.25 \\
\hline & $\mathrm{TT}$ & 0.13 & 0.12 & $0.83(0.52-1.32)$ & 0.43 \\
\hline \multirow{7}{*}{ KRAS rs61764370 } & $\mathrm{C}$ & 0.65 & 0.68 & & \\
\hline & $\mathrm{T}$ & 0.35 & 0.32 & $0.88(0.71-1.10)$ & 0.26 \\
\hline & $\mathrm{TT}$ & 0.93 & 0.95 & & \\
\hline & TG & 0.07 & 0.04 & $0.57(0.29-1.13)$ & 0.1 \\
\hline & GG & 0 & 0.01 & $13.3(0.71-248.1)$ & 0.06 \\
\hline & $\mathrm{T}$ & 0.97 & 0.97 & & \\
\hline & $\mathrm{G}$ & 0.03 & 0.03 & $0.97(0.55-1.72)$ & 0.91 \\
\hline
\end{tabular}

$\mathrm{OR}=$ odds ratio, $\mathrm{CI}=$ confidence interval. *After Bonferroni correction

Table 3. Genotype and Allele Frequencies after Gender Stratification

\begin{tabular}{|c|c|c|c|c|c|c|c|c|c|}
\hline \multirow{3}{*}{ Gene } & \multirow{3}{*}{$\begin{array}{c}\text { Genotype/ } \\
\text { Allele }\end{array}$} & \multicolumn{2}{|c|}{ Females } & \multirow{3}{*}{ OR } & \multirow{3}{*}{ Pvalue* } & \multicolumn{2}{|c|}{ Males } & \multirow{3}{*}{ OR } & \multirow{3}{*}{ Pvalue* } \\
\hline & & Control & CML & & & Control & CML & & \\
\hline & & $n=206$ & $n=137$ & & & $n=263$ & $n=175$ & & \\
\hline \multirow{5}{*}{$\begin{array}{c}\text { AGO1 } \\
\text { rs636832 }\end{array}$} & AA & 0.3 & 0.2 & & & 0.23 & 0.26 & & \\
\hline & AG & 0.43 & 0.5 & 1.73 & 0.19 & 0.53 & 0.5 & 0.85 & 0.49 \\
\hline & GG & 0.27 & 0.31 & 1.69 & 0.36 & 0.24 & 0.24 & 0.92 & 0.78 \\
\hline & $\mathrm{A}$ & 0.51 & 0.45 & & & 0.49 & 0.51 & & \\
\hline & $\mathrm{G}$ & 0.49 & 0.55 & 1.31 & 0.32 & 0.51 & 0.49 & 0.96 & 0.78 \\
\hline \multirow{5}{*}{$\begin{array}{c}\text { GEMIN4 } \\
\text { rs2740348 }\end{array}$} & GG & 0.64 & 0.68 & & & 0.6 & 0.63 & & \\
\hline & GC & 0.33 & 0.27 & 0.77 & 0.29 & 0.36 & 0.33 & 0.88 & 0.56 \\
\hline & $\mathrm{CC}$ & 0.03 & 0.05 & 1.65 & 0.37 & 0.04 & 0.04 & 0.91 & 0.86 \\
\hline & $\mathrm{G}$ & 0.81 & 0.81 & & & 0.78 & 0.79 & & \\
\hline & $\mathrm{C}$ & 0.19 & 0.19 & 0.95 & 0.79 & 0.22 & 0.21 & 0.91 & 0.6 \\
\hline \multirow{5}{*}{$\begin{array}{c}\text { BRCA1 } \\
\text { rs799917 }\end{array}$} & $\mathrm{CC}$ & 0.43 & 0.42 & & & 0.43 & 0.51 & & \\
\hline & $\mathrm{CT}$ & 0.42 & 0.46 & 1.1 & 0.69 & 0.47 & 0.38 & 0.67 & 0.24 \\
\hline & $\mathrm{TT}$ & 0.15 & 0.12 & 0.78 & 0.48 & 0.1 & 0.11 & 0.9 & 0.74 \\
\hline & $\mathrm{C}$ & 0.64 & 0.65 & & & 0.66 & 0.7 & & \\
\hline & $\mathrm{T}$ & 0.36 & 0.35 & 0.94 & 0.69 & 0.34 & 0.3 & 0.84 & 0.25 \\
\hline \multirow{5}{*}{$\begin{array}{c}\text { KRAS } \\
\text { rs61764370 }\end{array}$} & $\mathrm{TT}$ & 0.91 & 0.92 & & & 0.95 & 0.97 & & \\
\hline & TG & 0.09 & 0.05 & 0.58 & 0.23 & 0.05 & 0.03 & 0.57 & 0.28 \\
\hline & GG & 0 & 0.03 & 13.41 & 0.04 & 0 & 0 & 1.47 & 1 \\
\hline & $\mathrm{T}$ & 0.96 & 0.95 & & & 0.98 & 0.99 & & \\
\hline & $\mathrm{G}$ & 0.04 & 0.05 & 1.27 & 0.51 & 0.02 & 0.01 & 0.57 & 0.29 \\
\hline
\end{tabular}

$\mathrm{OR}=$ odds ratio, $\mathrm{CI}=$ confidence interval. $*$ After Bonferroni correction

BRCA1_rs799917, and KRAS_rs61764370 in our sample population, we found them to be in Hardy-Weinberg equilibrium in both cases and controls. Regarding the case-control study, the minor homozygote genotype from the KRAS_rs61764370 SNP (G/G) showed a borderline association with CML $(\mathrm{P}=0.06)$. Nevertheless, when we stratified by gender the minor homozygote genotype of this SNP showed a significantly higher frequency in women with $\mathrm{CML}(\mathrm{OR}=13.41 ; \mathrm{P}=0.04)$, but not in men (Tables 2 and 3). In fact, it is worth noting that in our entire population, we found only four individuals with the minor homozygote genotype, all of them were women affected by CML (Tables 2 and 3). Otherwise, the distribution of the allelic and genotypic frequencies of the AGO1_rs636832, GEMIN4_rs2740348, and BRCA1_rs799917 SNPs was similar between cases and controls, even after gender stratification (Tables 2 and 3).

Additionally, we compared the genotypic and allelic frequency of the four studied SNPs in patients in the chronic phase of CML (early stage) to those of patients whose disease was in the accelerated or blastic phases (late stage). We observed that the genotype and the allele frequencies of the four SNPs were similar between individuals in the early stage of the disease relative to those 
Table 4. CML Progression and SNPs Located in AGO1, BRCA1, GEMIN4 and KRAS Genes

\begin{tabular}{|c|c|c|c|c|c|}
\hline Gene & $\begin{array}{c}\text { Genotype/ } \\
\text { Allele }\end{array}$ & $\begin{array}{c}\mathrm{CP} \\
\mathrm{n}=98\end{array}$ & $\begin{array}{l}\mathrm{AP} / \mathrm{BP} \\
\mathrm{n}=27\end{array}$ & OR ( $95 \% \mathrm{CI})$ & Pvalue* \\
\hline \multirow{5}{*}{ AGO1 rs636832 } & $\mathrm{AA}$ & 0.26 & 0.15 & & \\
\hline & AG & 0.48 & 0.63 & $2.26(0.69-7.45)$ & 0.17 \\
\hline & GG & 0.26 & 0.22 & $1.50(0.38-5.97)$ & 0.56 \\
\hline & A & 0.5 & 0.46 & & \\
\hline & $\mathrm{G}$ & 0.5 & 0.54 & $1.16(0.63-2.12)$ & 0.63 \\
\hline \multirow{5}{*}{ GEMIN4 rs2740348 } & GG & 0.66 & 0.66 & & \\
\hline & GC & 0.28 & 0.3 & $1.07(0.42-2.76)$ & 0.89 \\
\hline & $\mathrm{CC}$ & 0.06 & 0.04 & $0.60(0.07-5.33)$ & 0.64 \\
\hline & $\mathrm{G}$ & 0.8 & 0.81 & & \\
\hline & $\mathrm{C}$ & 0.2 & 0.19 & $0.92(0.42-1.98)$ & 0.82 \\
\hline \multirow{5}{*}{ BRCA1 rs799917 } & $\mathrm{CC}$ & 0.42 & 0.52 & & \\
\hline & $\mathrm{CT}$ & 0.4 & 0.48 & $0.98(0.41-2.34)$ & 0.95 \\
\hline & TT & 0.18 & 0 & $0.08(0.00-1.37)$ & 0.1 \\
\hline & $\mathrm{C}$ & 0.62 & 0.76 & & \\
\hline & $\mathrm{T}$ & 0.38 & 0.24 & $0.51(0.26-1.02)$ & 0.19 \\
\hline \multirow{5}{*}{ KRAS rs61764370 } & TT & 0.95 & 0.85 & & \\
\hline & TG & 0.04 & 0.07 & $2.02(0.35-11.72)$ & 0.42 \\
\hline & GG & 0.01 & 0.07 & $8.09(0.70-93.10)$ & 0.19 \\
\hline & $\mathrm{T}$ & 0.97 & 0.89 & & \\
\hline & $\mathrm{G}$ & 0.03 & 0.11 & $3.96(1.22-12.82)$ & 0.16 \\
\hline
\end{tabular}

$\mathrm{OR}=$ odds ratio, $\mathrm{CI}=$ confidence interval; $\mathrm{CP}$ : chronic phase; $\mathrm{AP} / \mathrm{BP}$ : accelerated phase and blast phase. *After Bonferroni correction

Table 5. Therapeutic Response to Imatinib Treatment

\begin{tabular}{|c|c|c|c|c|c|}
\hline Gene & Genotype/Allele & $\begin{array}{c}\text { Responsive } \\
\mathrm{n}=66\end{array}$ & $\begin{array}{c}\text { Resistant } \\
\mathrm{n}=62\end{array}$ & OR $(95 \% \mathrm{CI})$ & Pvalue* \\
\hline \multirow{5}{*}{ AGO1 rs636832 } & AA & 0.24 & 0.23 & & \\
\hline & $\mathrm{AG}$ & 0.45 & 0.56 & $1.33(0.56-3.17)$ & 0.51 \\
\hline & GG & 0.3 & 0.21 & $0.74(0.27-2.02)$ & 0.56 \\
\hline & A & 0.47 & 0.51 & & \\
\hline & $\mathrm{G}$ & 0.53 & 0.49 & $0.86(0.52-1.40)$ & 0.54 \\
\hline \multirow{5}{*}{ GEMIN4 rs2740348 } & GG & 0.64 & 0.66 & & \\
\hline & GC & 0.32 & 0.29 & $0.88(0.41-1.88)$ & 0.74 \\
\hline & $\mathrm{CC}$ & 0.05 & 0.05 & $1.02(0.19-5.37)$ & 0.98 \\
\hline & $\mathrm{G}$ & 0.8 & 0.81 & & \\
\hline & $\mathrm{C}$ & 0.2 & 0.19 & $0.93(0.50-1.72)$ & 0.82 \\
\hline \multirow{5}{*}{ BRCA1 rs799917 } & $\mathrm{CC}$ & 0.41 & 0.4 & & \\
\hline & $\mathrm{CT}$ & 0.38 & 0.52 & $1.38(0.65-2.94)$ & 0.4 \\
\hline & $\mathrm{TT}$ & 0.21 & 0.08 & $0.39(0.12-1.23)$ & 0.1 \\
\hline & $\mathrm{C}$ & 0.6 & 0.66 & & \\
\hline & $\mathrm{T}$ & 0.4 & 0.34 & $0.76(0.46-1.27)$ & 0.3 \\
\hline \multirow{5}{*}{ KRAS rs61764370 } & $\mathrm{TT}$ & 0.93 & 0.92 & & \\
\hline & TG & 0.05 & 0.06 & $1.45(0.31-6.76)$ & 0.63 \\
\hline & GG & 0.02 & 0.02 & $1.09(0.07-17.80)$ & 0.95 \\
\hline & $\mathrm{T}$ & 0.96 & 0.95 & & \\
\hline & $\mathrm{G}$ & 0.04 & 0.05 & $1.29(0.38-4.34)$ & 0.68 \\
\hline
\end{tabular}

$\mathrm{OR}=$ odds ratio, $\mathrm{CI}=$ confidence interval. $*$ After Bonferroni correction

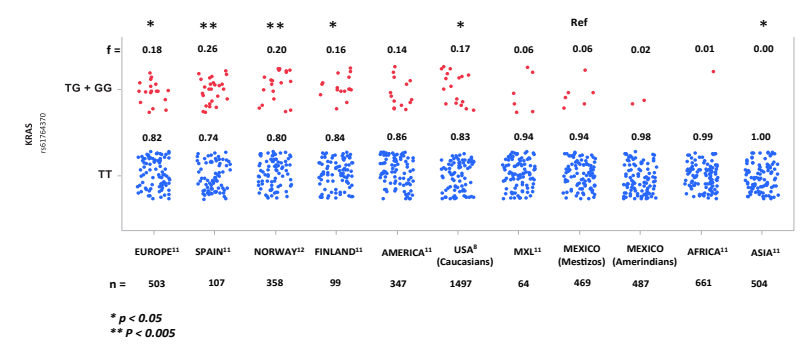

Figure 1. Frequency of SNP rs61764370 $(T>G)$ of KRAS Gene in Different Populations Worldwide, Ref= Reference Population in the late stage (Table 4). We also analyzed the association of these gene variants with the response to imatinib as CML therapy, but the distribution of the genotype and allele frequency was similar between patients with resistant versus responsive disease (Table 5).

Because of the impact of the KRAS_rs61764370 SNP in Mexican CML women, we determined the prevalence of this variant in a sample of healthy Amerindian individuals. We found a significantly lower frequency of the KRAS_rs61764370 variant in healthy Amerindians than in Mestizo individuals (1\% vs. 4\%), and still lower than those reported in different Caucasian populations 
such as Spain, Norway, Finland and USA (14\%, 10\%, $9 \%$ and $9 \%$, respectively) (Figure 1) (Chin et al., 2008; Genomes Project et al., 2012; Kjersem et al., 2012).

\section{Discussion}

Because miRNAs are central regulators of cellular processes such as proliferation, differentiation, and apoptosis, mutations affecting genes coding for miRNAs are commonly found in several types of human tumors. Recent compelling evidence indicates that functional SNPs occurring at MBS or in genes involved in the miRNA biogenesis pathway may modify cancer predisposition.

Here, we analyzed as susceptibility factors for CML the SNPs rs61764370 and rs799917, located in the MBS of the KRAS oncogene and BRCA1 tumor suppressor gene, respectively as well as the SNPs rs636832 and rs2740348 in AGO1 and GEMIN4, respectively, both genes involved in miRNA biogenesis. To the best of our knowledge, this study is the first to evaluate the association of AGO1_ rs636832, GEMIN4_rs2740348, BRCA1_rs799917, and KRAS_rs61764370 variants with CML.

We determined that the minor homozygote genotype of the KRAS_rs61764370 SNP had a borderline association with CML susceptibility $(\mathrm{P}=0.06)$ that became significant among women after gender stratification $(\mathrm{OR}=13.41$; $\mathrm{P}=0.04$ ). In fact, we found only four individuals carrying the minor homozygote genotype for this SNP in our entire population $(n=781)$. Of interest, these four individuals were women, and all of them had CML. This finding may indicate a gender-specific association of the KRAS_rs61764370 variant with CML. Because of the heterogeneity in the allele distribution of the KRAS rs61764370 variant among populations, we also analyzed the frequency of this allele in Amerindians, showing that this population has one of the lowest frequencies worldwide and suggesting that this variant was provided to the Mestizos from European populations.

The rs61764370 SNP is located in the 3'UTR region of the KRAS oncogene, and its derived allele has been associated with an increased expression of KRAS by weakening the microRNA let-7 binding to KRAS mRNA (Chin et al., 2008). This variant has been associated with an increased risk of triple-negative breast cancer, non-small cell lung cancer, and ovarian cancer (Chin et al., 2008; Ratner et al., 2010; Paranjape et al., 2011). The GTPase protein encoded by KRAS, and the miRNA let- 7 are involved in the regulation of proliferation, differentiation, and apoptosis. In fact, let-7 is a master regulator in the differentiation process of normal hematopoietic stem cells (Copley et al., 2013), while activating mutations in KRAS are one of the most common events in cancer development (Samatar and Poulikakos, 2014). It is worth noting that point mutations in KRAS are frequent in acute myeloid and lymphoblastic leukemias and that loss of inhibit ory regulators of this oncoprotein drives a murine model of myeloid leukemogenesis (Chandra et al., 2010; Knight and Irving, 2014; Zhao et al., 2015). Collectively with our results, these data suggest that KRAS and let-7 are important molecules in leukemogenesis and that the KRAS_rs61764370 genotype may influence individual susceptibility to CML development. However, because of the small size and the low frequency of this allele, further analyses are needed to confirm this finding.

Gender-specific association of risk alleles with complex diseases has been reported previously. For instance, minor homozygote genotype of rs2304456 and rs4686799 SNPs of the kininogen 1 gene was associated with essential hypertension in male population, whereas the minor allele of rs362719 SNP of the RELN gene was associated with schizophrenia in female population (Zhao et al., 2009; Kuang et al., 2011). It has been suggested that gender-specific differences in gene expression regulation may underlie gender-dependent susceptibility to complex diseases (Ober et al., 2008; Dimas et al., 2012). Further studies are warranted to evaluate the expression levels of Let-7 in male and female individuals affected by CML.

On the other hand, we observed no significant differences in the genotype and allele distribution of the SNPs AGO1_rs636832, GEMIN4_rs2740348, or BRCA1_rs799917 among healthy individuals and subjects affected by CML, even after gender stratification.

In spite that these genes have been consistently involved in cancer, the role of these SNPs in the oncogenesis process remains still controversial. The BRCA1_rs799917 SNP is located in the coding sequence of the gene and alters its expression by affecting the interaction of miR-638 with BRCA1 mRNA (Nicoloso et al., 2010; Zhang et al., 2013). This SNP has been associated with an increased risk of breast, gastric, and esophageal cancer (Nicoloso et al., 2010; Zhang et al., 2013; Wang et al., 2015). Regarding the AGO1_rs636832 gene variant, it has been involved with reduced expression of the gene, and associated with a decreased risk of lung cancer (Nicoloso et al., 2010; Zhang et al., 2013; Wang et al., 2015). Finally, GEMIN4_ rs2740348 is a nonsynonymous variation (Gln439Glu) that may reduce the enzymatic activity of the protein and it has been associated with a high risk of prostate cancer but a reduced risk of renal carcinoma as part of a common haplotype of GEMIN4 (Horikawa et al., 2008; Kim et al., 2010; Liu et al., 2012). The conflicting results obtained with these gene variants could result from ethnic differences, a cancer-specific effect of the SNP, or small study samples but also could indicate the involvement of other genetic and environmental factors in the effect of the gene variants in cancer susceptibility.

In conclusion, we found a significant association of the homozygous GG genotype of the KRAS_rs61764370 variant with a higher risk for developing CML in a gender-dependent manner. Because ethnic ancestry is related to SNP frequency, the association of this SNP on the KRAS gene with CML must be validated in different ethnic groups.

\section{Acknowledgements}

GMH is a Ph.D. student from the Posgrado en Ciencias Biológicas, Universidad Nacional Autónoma de México, México, and recipient of a fellowship from CONACyT (220196). This study was conducted as part of his doctoral thesis. We would like to acknowledge to Dr. Marcela Varela Gómez, IBQ Julio Cesar Canseco M, and Biol. 
Humberto Gutierrez-Malacatt et al

Alfredo Mendoza-Vargas from the USec INMEGEN for technical support, and Guillermo Escamilla-Guerrero for control sample collecting.

\section{References}

Apperley JF (2015). Chronic myeloid leukaemia. Lancet, 385, 1447-59.

Copley MR, Babovic S, Benz C, et al (2013). The Lin28b-let-7Hmga2 axis determines the higher self-renewal potential of fetal haematopoietic stem cells. Nat Cell Biol, 15, 916-25.

Chandra P, Luthra R, Zuo Z, et al (2010). Acute myeloid leukemia with $\mathrm{t}(9 ; 11)(\mathrm{p} 21-22 ; \mathrm{q} 23)$ : common properties of dysregulated ras pathway signaling and genomic progression characterize de novo and therapy-related cases. Am J Clin Pathol, 133, 686-93.

Chin LJ, Ratner E, Leng S, et al (2008). A SNP in a let-7 microRNA complementary site in the KRAS 3' untranslated region increases non-small cell lung cancer risk. Cancer Res, 68, 8535-40.

Dimas AS, Nica AC, Montgomery SB, et al (2012). Sex-biased genetic effects on gene regulation in humans. Genome Res, 22, 2368-75.

Genomes Project C, Abecasis GR, Auton A, et al (2012). An integrated map of genetic variation from 1,092 human genomes. Nature, 491, 56-65.

Ha M, Kim VN (2014). Regulation of microRNA biogenesis. Nat Rev Mol Cell Biol, 15, 509-24.

Horikawa Y, Wood CG, Yang H, et al (2008). Single nucleotide polymorphisms of microRNA machinery genes modify the risk of renal cell carcinoma. Clin Cancer Res, 14, 7956-62.

Kim JS, Choi YY, Jin G, et al (2010). Association of a common AGO1 variant with lung cancer risk: a two-stage case-control study. Mol Carcinog, 49, 913-21.

Kjersem JB, Ikdahl T, Guren T, et al (2012). Let-7 miRNAbinding site polymorphism in the KRAS 3'UTR; colorectal cancer screening population prevalence and influence on clinical outcome in patients with metastatic colorectal cancer treated with 5-fluorouracil and oxaliplatin $+/$ - cetuximab. BMC Cancer, 12, 534.

Knight T, Irving JA (2014). Ras/Raf/MEK/ERK Pathway Activation in Childhood Acute Lymphoblastic Leukemia and Its Therapeutic Targeting. Front Oncol, 4, 160.

Kuang WJ, Sun RF, Zhu YS, et al (2011). A new singlenucleotide mutation (rs362719) of the reelin (RELN) gene associated with schizophrenia in female Chinese Han. Genet Mol Res, 10, 1650-8.

Liu J, Liu J, Wei M, et al (2012). Genetic variants in the microRNA machinery gene GEMIN4 are associated with risk of prostate cancer: a case-control study of the Chinese Han population. DNA Cell Biol, 31, 1296-302.

Nicoloso MS, Sun H, Spizzo R, et al (2010). Single-nucleotide polymorphisms inside microRNA target sites influence tumor susceptibility. Cancer Res, 70, 2789-98.

Ober C, Loisel DA, Gilad Y (2008). Sex-specific genetic architecture of human disease. Nat Rev Genet, 9, 911-22.

Paranjape T, Heneghan H, Lindner R, et al (2011). A 3'-untranslated region KRAS variant and triple-negative breast cancer: a case-control and genetic analysis. Lancet Oncol, 12, 377-86.

Radich J (2014). Structure, function, and resistance in chronic myeloid leukemia. Cancer Cell, 26, 305-6.

Ratner E, Lu L, Boeke M, et al (2010). A KRAS-variant in ovarian cancer acts as a genetic marker of cancer risk. Cancer Res, 70, 6509-15.

Samatar AA, Poulikakos PI (2014). Targeting RAS-ERK signalling in cancer: promises and challenges. Nat Rev Drug
Discov, 13, 928-42.

Shang M, Huang Y, Hu X, et al (2014). Association between SNPs in miRNA-machinery genes and chronic hepatitis B in the Chinese Han population. Infect Genet Evol, 28, 113-7.

Ursan ID, Jiang R, Pickard EM, et al (2015). Emergence of BCR-ABL kinase domain mutations associated with newly diagnosed chronic myeloid leukemia: a meta-analysis of clinical trials of tyrosine kinase inhibitors. J Manag Care Spec Pharm, 21, 114-22.

Wang K, Xu L, Pan L, et al (2015). The functional BRCA1 rs799917 genetic polymorphism is associated with gastric cancer risk in a Chinese Han population. Tumour Biol, 36, 393-7.

Zhang X, Wei J, Zhou L, et al (2013). A functional BRCA1 coding sequence genetic variant contributes to risk of esophageal squamous cell carcinoma. Carcinogenesis, 34, 2309-13.

Zhao W, Wang Y, Wang L, et al (2009). Gender-specific association between the kininogen 1 gene variants and essential hypertension in Chinese Han population. $J$ Hypertens, 27, 484-90.

Zhao Z, Chen CC, Rillahan CD, et al (2015). Cooperative loss of RAS feedback regulation drives myeloid leukemogenesis. Nat Genet, 47, 539-43. 